\title{
Rederivation of transgenic and gene-targeted mice by embryo transfer
}

\author{
Margaret L. Van Keuren ${ }^{1} \&$ Thomas L. Saunders ${ }^{1,2, *}$ \\ ${ }^{1}$ Transgenic Animal Model Core, ${ }^{2}$ Department of Internal Medicine, Division of Molecular Medicine and Genetics, \\ University of Michigan Medical School, 2560 MSRB 11 Box 0674, 1150 w.med.center Dr. Ann Arbor, MI \\ 48109, USA
}

Received 6 November 2003; revised 23 March 2004; accepted 31 March 2004

Key words: embryo transfer, mouse virus, mutation, pathogen , rederivation, transgenic mice

\begin{abstract}
Research on genetically engineered mice provides insights into the etiology, therapy, and genetic basis of human diseases. An important variable that affects the results of mouse studies is the health status of the animals. Pathogen burdens may confound observations and obscure underlying mechanisms. Mouse resource centers frequently rederive infected mouse strains. We review our experience on the use of a well-established technique, embryo transfer to rederive infected mouse strains. The following mouse pathogens were eliminated by embryo transfer: Mouse Parvovirus, Mouse Hepatitis Virus, Mouse Rotavirus, Mouse Encephalomyelitis Virus, Mouse Adenovirus, Helicobacter species, endoparasites, and ectoparasites. We rederived transgenic mouse lines, gene-targeted mouse lines, and lines with spontaneous mutations. In the majority of strains, fertilized eggs for embryo transfer were obtained by mating superovulated egg donors with males of the desired genotype. A total of 309 embryo transfers were performed to rederive 96 mouse strains. The pregnancy rate was $76 \%$; 1996 pups were born, of which $43 \%$ carried the desired genotype. We performed 44 additional embryo transfers to rederive 15 other strains. The pregnancy rate was lower (45\%) and none of the 135 pups carried the desired genotype. Although we successfully eliminated the pathogens in all transfers, we were unable to obtain pups with the desired genotype in 15 of 111 mouse lines. Multiple factors affect the efficiency of rederivation by embryo transfer. They include the response to superovulation by embryo donors, the number and age of stud males, the yield of fertilized eggs, the number of embryo transfers, and genotyping.
\end{abstract}

\section{Introduction}

Use of genetically engineered mice to understand human disease processes is increasing. It is important that these studies are conducted in specific pathogen free (SPF) mouse models. Animals need to be free of pathogen infections if physiological changes attributed to genetic alterations are to be extrapolated to other species. If the mouse models are pathogen infected their response to infection may mask genetic changes

*Author for correspondence E-mail: tsaunder@umich.edu and/or complicate the interpretation of results. Viral, bacterial, and parasitic infections can interfere with research results in animal models (National Research Council, 1991, reviewed in Homberger et al., 1999). Consequently, it is frequently desirable to rederive mouse stocks to SPF status. This is often required to import animals into clean barrier facilities, to replace populations after a spontaneous infection of unknown etiology, or to eliminate pathogens that may interfere with research. We have extensive experience in pathogen free rederivation of mouse stocks. In our hands, the use of embryo transfer is a reliable and effective method of rederivation. 
The pathophysiological response to infection may obscure the consequences of genetic alterations. For example, viral infections cause immune system dysfunction (Jacoby et al., 1996; Homberger 1997). In addition to provoking physiological changes, the clinical presentation of viral infections can mimic phenotypes caused by experimental genetic changes in mice. For example, hindlimb paralysis occurs in alpha A-crystallin transgenic mice (De Rijk et al., 2000) and in mice infected with Murine Hepatitis Virus (MHV) (Glass et al., 2002) or Theilers Mouse Encephalomyelitis Virus (TMEV) (Tsunoda et al., 1996). In some cases, an infection is responsible for producing a phenotype in a gene-targeted mouse that would otherwise appear normal. Engle et al. (2002) reported that double mutant mice for Tgfb1 and Rag2 develop colitis and colon cancer if they are infected with Helicobacter hepaticus but remain free of inflammatory lesions and carcinoma in the absence of $H$. hepaticus organisms. Sensitive behavioral tests are also influenced by experimental viral infections. Reduced activity in open-field behavior tests occurred in v-Ha-ras transgenic mice (Colombo et al., 2000) and in mice experimentally infected with human influenza virus (Shi et al., 2003). Just as the presence of a pathogen can induce a phenotypic alteration, so can the treatment of mice for parasites. A common approach to pinworm infection is to treat mice with the anti-helmitic drug Ivermectin. Paradoxically, although this treatment effectively eliminates pinworm and its consequences, Ivermectin treatment alters the outcome of mouse behavior tests (Davis et al., 1999). Research on physiological changes attributed to experimentally induced genetic changes needs to be distinct from changes induced by pathogen infections. Careful health assessment of these animals is required to determine whether changes are due to genetic manipulation.

Rederivation of pathogen-infected mice by embryo transfer to pathogen free recipients is an effective method of disease elimination. Reetz et al., (1988) eliminated MHV and Pasteurella pneumotropica from six inbred strains. Morrel (1999) reported the elimination of MHV, rotavirus (Epizootic Diarrhea of Infant Mice Virus: EDIM), reo-3 virus, TMEV, and Mouse Adenovirus (MAD). Selecting embryos with intact zona pellucida (z.p.) for embryo transfer is important because the z.p. prevents contact between infectious viral particles and the cell membrane. Incubation of eggs with and without z.p. in Mouse Parvovirus (MPV) suspensions demonstrated that the z.p. prevents infection from occurring (Mohanty \& Bachman, 1974). Similarly, both Reetz et al. (1988) and Carthew et al. (1985) incubated embryos with or without z.p. in MHV suspensions and observed that the z.p. prevents MHV infection. Indirect evidence suggests that the z.p. also protects embryos from TMEV since inoculation of pregnant female mice with the virus on days 1-3 of gestation (while preimplantation embryos are protected by the z.p.) does not result in virus positive fetuses although positive fetuses are found when virus is inoculated on gestational days 4 and 5 (after the z.p. is shed) (Abzug \& Tyson, 2000). Hill and Stalley (1991) showed that Mycoplasma pulmonis could be eliminated from z.p. intact eggs by overnight culture in mouse embryo media, which does not support M. pulmonis growth. Serial dilution of preimplantation embryos collected from infected mice reduces the concentration of infectious organisms below the threshold of infection in the transfer fluid (Stringfellow, 1998). As a consequence, pups, born after transfer of washed embryos to SPF surrogate mothers, enjoy the same pathogen free status as their mothers.

We summarize our results on the effectiveness of mouse embryo transfer to rederive 111 genetically altered mouse strains with enzootic pathogen infections. Our results confirm the elimination of MHV, EDIM, TMEV, and MAD by embryo transfer. In addition, we show that this method effectively eliminates MPV, Helicobacter spp., M. pulmonis, endoparasites (pinworm), and ectoparasites (fur mites). We discuss our success rate and the factors that influence it. We compare embryo transfer rederivation with alternatives.

\section{Methods and materials}

\section{Animals}

Adult (6 weeks to 6 months old) (C57BL/6J X DBA/2J) F1 male and female mice were obtained from the Jackson Laboratory (Bar Harbor, Maine). Adult CD-1 male and female mice were obtained from Charles River Laboratory (Wilmington, Delaware). We used CD-1 mice to 
produce pseudopregnant recipients until year 2000 and used (C57BL/6J X DBA/2J)F1 mice since then. Vasectomized males were housed one per cage and females were housed five per cage prior to embryo transfer. Vasectomized males were mated randomly with two or three females naturally cycling through estrus to produce pseudopregnant recipients for embryo transfers. Weanling ( 3 weeks old) egg donors $(\mathrm{C} 57 \mathrm{BL} / 6 \mathrm{~J}$, $\mathrm{C} 3 \mathrm{H} / \mathrm{HeJ}$, DBA $/ 2 \mathrm{~J}$, and $129 \mathrm{~S} 1 / \mathrm{Sv} / \mathrm{mJ})$ were obtained from the Jackson Laboratory. Egg donors were superovulated and mated with infected males to produce fertilized mouse eggs. If there was a need to maintain the unique mixed genetic background of a mouse strain then both egg donors and stud males were obtained from the infected mouse colony. Static microisolater caging (Allentown Caging, Allentown, New Jersey) and ground corncob bedding were used for all animals. Access to water and food was ad libitum. The animals were fed Purina 5008 rodent diet. Animal rooms were climate controlled to provide temperatures of $22-23^{\circ} \mathrm{C}$ and relative humidity of $15 \%$ on a 12-h light/dark cycle (lights on at 0600). All procedures were approved by the University of Michigan Committee on Use and Care of Animals. Animal care was provided in accordance with the principles and procedures outlined in the National Research Council Guide for the Care and Use of Laboratory Animals.

\section{Egg collection and washing}

Superovulation was used to increase the numbers of eggs per donor and to reduce the total number of egg donors required. At 15:00, egg donors were injected intraperitoneally with 5 I.U. pregnant mare's serum gonadotropin obtained from the National Hormone and Peptide Program (National Institute of Diabetes and Digestive and Kidney Diseases). After $46 \mathrm{~h}$, egg donors were injected intraperitoneally with 5 I.U. of human chorionic gonadotropin (HCG, Sigma Chemical Company). After HCG treatment, egg donors were taken from their pathogen free mouse room to the quarantine room and singly mated with infected stud males. Whenever possible, 3-6 males were mated so that there were enough fertilized eggs for transfer to multiple recipients.
The following morning (09:00-11:00) egg donors were euthanized in the quarantine room and submerged in $3.3 \%$ bleach during transportation to the egg collection laboratory (5-8 mins). This treatment is expected to inactivate viruses, bacteria, parasites and their eggs (D. Ringler, personal communication). The eggs were stripped of cumulus granulosa cells by hyaluronidase treatment as described (Hogan et al. 1994). Fertilized eggs with intact zona pellucida were selected for subsequent washing and transfer. Eggs were sequentially washed five times in $1.0 \mathrm{ml}$ drops of sterile M2 medium (Sigma Chemical Co., St. Louis, Missouri) with changes of sterile transfer pipets between drops. Eggs were washed through four drops of $0.075 \mathrm{ml} \mathrm{C0} 0_{2}$ equilibrated BOMC medium (Invitrogen, Carlsbad, California) supplemented with 5000 I.U./ml penicillin and streptomycin (Invitrogen) with transfer pipet changes between drops. The eggs were incubated in BOMC at $37^{\circ} \mathrm{C}, 5 \% \mathrm{CO}_{2}, 95 \%$ relative humidity until surgical transfer. The washing steps diluted the original volume of fluid containing the eggs by a factor of $1 \times 10^{21}$, which exceeds the $1 \times 10^{20}$ dilution, recommended by the International Embryo Transfer Society (Stringfellow, 1998). For the elimination of Mycoplasma pulmonis, embryos were incubated overnight and transferred the following day (Hill \& Stalley, 1991).

\section{Embryo transfer and genotyping}

Pseudopregnant females were identified by the presence of a copulation plug after mating with vasectomized males. Embryo recipients were anesthetized with ketamine/xylazine as described by Zeller et al. (1998). Egg transfer into oviducts was performed as described in Hogan et al. (1994). Each recipient received 20-25 one-cell egg divided between the two oviducts. When eggs were cultured overnight, 16-20 two-cell eggs were divided between the oviducts. Recipients were housed one or two per cage. When pups were 2 weeks of age, they were ear tagged (National Band and Tag, Newport, Kentucky) for tracking purposes and 5-mm tail tip biopsies were collected for DNA extraction. Each DNA sample was genotyped by a PCR assay for a specific transgene or targeted gene. All genotyping assays were performed by the research laboratories that 
submitted animals for rederivation. When a genetic test was unavailable for the desired genetic background, offspring were bred to verify the transmission of the desired phenotype. This was often the case for mouse strains carrying spontaneous mutations that were molecularly uncharacterized or for which the genetic etiology had not been established.

\section{Pathogen testing}

Serum samples were collected from dams 6-8 weeks after the embryo transfer procedure. Immuncomb tests (Charles River Laboratory) were used on site (University of Michigan Unit for Laboratory Animal Medicine) to detect antibodies to MHV, Sendai virus, and Mycoplasma pulmonis. Cecal flotation was used to detect the presence of Aspicularis tetraptera (pinworm). Myobia musculi (fur mites) were detected by examining skin and fur. All other assays (MPV, EDIM, TMEV (GDVII), MAD, and Helicobacter spp.) were performed offsite as part of Health Assessment Plus testing at Charles River Laboratories.

\section{Criteria for success}

Three criteria were used to determine whether a mouse strain was successfully rederived as a SPF mouse line. (1) Viable animals of the desired genotype were produced. For transgenic or genetargeted mouse strains crossed to standard inbred mouse lines, at least one male or female pup with the transgene was produced. For strains in mixed genetic backgrounds, at least one male and one female pup were produced to establish a breeding pair. (2) Embryo recipients were pathogen free 6-8 weeks after embryo transfer. (3) Subsequent surveillance of pups and their offspring demonstrated they continued to be pathogen free.

\section{Results}

\section{MHV elimination}

Over a 3-year period 42 embryo transfers were performed to successfully eliminate MHV from nine mouse strains backcrossed to C57BL/6 (two gene-targeted and seven transgenic lines). Fertilized eggs were obtained for transfer by mating pathogen free $\mathrm{C} 57 \mathrm{BL} / 6$ females with infected stud males. In the successful rederivations, $81 \%$ of the embryo recipients became pregnant and delivered an average of seven pups (Table 1). MHV was eliminated in every case; however, pups with the desired genotype were not obtained from three strains.

\section{MPV elimination}

Over a 2-year period, 77 embryo transfers were performed to eliminate MPV from 47 mouse strains. One of these was a gene-targeted strain on a mixed 129/Sv genetic background, the other 46 were transgenic strains backcrossed to $\mathrm{C} 57 \mathrm{BL} / 6$. Fertilized eggs were obtained by mating commercially procured pathogen-free C $57 \mathrm{BL} / 6$ females to infected transgenic males. In order to maintain the unique mixed genetic background of the genetargeted strain, infected embryo donors were superovulated and mated with infected stud males to obtain fertilized eggs. In the successful rederivations, $84 \%$ of the embryo recipients became pregnant and delivered an average of 10 pups (Table 2). MPV was eliminated in every case; however, pups with the desired genotype were not obtained from nine of the mouse strains.

\section{Elimination of Helicobacter species}

We rederived two mouse strains that were infected with Helicobacter species, as detected by diagnostic testing at Charles River Laboratory.

Table 1. Mouse hepatitis virus elimination by mouse embryo transfer

\begin{tabular}{llllll}
\hline Egg donor strain & $\begin{array}{l}\text { Strains } \\
\text { rederived }\end{array}$ & $\begin{array}{l}\text { Embryo transfer } \\
\text { recipients }\end{array}$ & $\begin{array}{l}\text { Number of } \\
\text { pregnancies }\end{array}$ & Number of pups born & $\begin{array}{l}\text { Pups with desired } \\
\text { genotype }\end{array}$ \\
\hline $\begin{array}{l}\text { C57BL/6J } \\
\text { Unsuccessful rederivation attempts }\end{array}$ & 92 & $34(81 \%)$ & 252 & $102(40 \%)$ \\
C57BL/6J & 3 & 16 & $7(44 \%)$ & 41 & $0(0 \%)$ \\
\hline
\end{tabular}


Table 2. Mouse parvovirus elimination by mouse embryo transfer

\begin{tabular}{|c|c|c|c|c|c|}
\hline Egg donor strain & $\begin{array}{l}\text { Strains } \\
\text { rederived }\end{array}$ & $\begin{array}{l}\text { Embryo transfer } \\
\text { recipients }\end{array}$ & $\begin{array}{l}\text { Number of } \\
\text { pregnancies }(\%)\end{array}$ & $\begin{array}{l}\text { Number of } \\
\text { pups born }\end{array}$ & $\begin{array}{l}\text { Pups with desired } \\
\text { genotype }(\%)\end{array}$ \\
\hline $\mathrm{C} 57 \mathrm{BL} / 6 \mathrm{~J}$ & 46 & 72 & $61(85 \%)$ & 610 & $225(37 \%)$ \\
\hline Mixed & 1 & 5 & $4(80 \%)$ & 55 & $36(65 \%)$ \\
\hline Combined Total & 47 & 77 & $65(84 \%)$ & 665 & $261(39 \%)$ \\
\hline \multicolumn{6}{|c|}{ Unsuccessful rederivation attempts } \\
\hline $\mathrm{C} 57 \mathrm{BL} / 6 \mathrm{~J}$ & 9 & 17 & $12(71 \%)$ & 93 & $0(0 \%)$ \\
\hline
\end{tabular}

One strain was a transgenic line on a mixed genetic background and the other was a spontaneous mutation in $\mathrm{C} 57 \mathrm{BL} / 6$. In order to maintain their unique genetic backgrounds, infected embryo donors were superovulated and mated with infected stud males to obtain fertilized eggs for embryo transfer for both strains. Fertilized eggs were transferred to 12 recipients resulting in six pregnancies. A total of 48 pups were born. Since egg donors and male studs were from the same genetic background, all of the pups born carried the desired genotype. Thus, embryo transfer succeeded in the rederivation of both mouse strains that were positive for Helicobacter species.

\section{Elimination of pinworm}

We rederived three mouse strains with pinworm (Aspicularis tetraptera) infections, as diagnosed by flotation of cecal contents. One was a genetargeted strain backcrossed to $129 \mathrm{~S} 1 / \mathrm{SvImJ}$ : the second was a gene-targeted strain backcrossed to $\mathrm{C} 57 \mathrm{BL} / 6$ and the third was a transgenic strain backcrossed to $\mathrm{C} 57 \mathrm{BL} / 6$. Fertilized eggs were transferred to 19 recipients resulting in sixteen pregnancies (84\%) (Table 3). A total of 127 pups were born for an average of eight pups per dam; 51 of the pups had the desired genotype. Thus, embryo transfer successfully eliminated pinworm infections in all three lines.

\section{Combined MHV, EDIM, and mite infections}

We performed 154 embryo transfers to rederive 32 mouse strains that were infected with two viruses and an ectoparasite (Table 4). The founder colonies were seropositive for MHV and EDIM. In addition, inspection of pelage demonstrated the

Table 3. Mouse pinworm elimination by mouse embryo transfer

\begin{tabular}{llllrr}
\hline Egg donor strain & $\begin{array}{l}\text { Strains } \\
\text { rederived }\end{array}$ & $\begin{array}{l}\text { Embryo transfer } \\
\text { recipients }\end{array}$ & $\begin{array}{l}\text { Number of } \\
\text { pregnancies (\%) }\end{array}$ & $\begin{array}{l}\text { Number of } \\
\text { pups born }\end{array}$ & $\begin{array}{l}\text { Pups with desired } \\
\text { genotype }(\%)\end{array}$ \\
\hline C57BL/6J & 2 & 12 & $11(92 \%)$ & 80 & $32(40 \%)$ \\
$129 \mathrm{~S} 1 /$ SvlmJ & 1 & 7 & $5(71 \%)$ & 47 & $19(40 \%)$ \\
Combined total & 3 & 19 & $16(84 \%)$ & 127 & $51(40 \%)$ \\
\hline
\end{tabular}

Table 4. Multiple pathogen elimination by mouse embryo transfer

\begin{tabular}{|c|c|c|c|c|c|}
\hline Egg donor strain & $\begin{array}{l}\text { Strains } \\
\text { rederived }\end{array}$ & $\begin{array}{l}\text { Embryo transfer } \\
\text { recipients }\end{array}$ & $\begin{array}{l}\text { Number of } \\
\text { pregnancies }(\%)\end{array}$ & $\begin{array}{l}\text { Number of } \\
\text { pups born }\end{array}$ & $\begin{array}{l}\text { Pups with desired } \\
\text { genotype }(\%)\end{array}$ \\
\hline $\mathrm{C} 57 \mathrm{BL} / 6 \mathrm{~J}$ & 28 & 138 & $98(71 \%)$ & 865 & $403(47 \%)$ \\
\hline $\mathrm{C} 3 \mathrm{H}$ & 1 & 6 & $3(50 \%)$ & 18 & $10(56 \%)$ \\
\hline CD-1 & 1 & 3 & $2(67 \%)$ & 11 & $5(45 \%)$ \\
\hline Mixed & 2 & 7 & $6(86 \%)$ & 58 & $25(43 \%)$ \\
\hline Combined total & 32 & 154 & $109(71 \%)$ & 952 & $443(46 \%)$ \\
\hline \multicolumn{6}{|c|}{ Unsuccessful rederivation attempts } \\
\hline $\mathrm{DBA} / 2$ & 2 & 8 & $1(12 \%)$ & 1 & $0(0 \%)$ \\
\hline Mixed & 1 & 3 & $0(0 \%)$ & 0 & $0(0 \%)$ \\
\hline
\end{tabular}


presence of fur mites. Twenty-eight strains were backcrossed to $\mathrm{C} 57 \mathrm{BL} / 6,15$ were transgenic, nine were spontaneous mutations, and four were genetargeted strains. One strain was a spontaneous mutant backcrossed to $\mathrm{C} 3 \mathrm{H} / \mathrm{HeJ}$, one was a transgenic on the CD-1 background and two were a spontaneous mutants on mixed genetic backgrounds. In order to maintain their unique genetic backgrounds, infected embryo donors from mixed genetic backgrounds were superovulated and mated with infected stud males to obtain fertilized eggs for embryo transfer. In the successful rederivations, $71 \%$ of the embryo recipients became pregnant and delivered an average of nine pups (Table 4). All three pathogens were eliminated in each case; however, no pups of the desired genotype were obtained from three strains.

Combined MHV, MPV, EDIM, MAD, GDVII, mycoplasma and pinworm infection

We rederived three mouse strains that were infected with multiple pathogens: MHV, MPV, EDIM, MAD, GDVII, Mycoplasma pulmonis, and pinworm. These three transgenic lines were on unique mixed genetic backgrounds so that infected embryo donors were superovulated and mated with infected stud males to obtain fertilized eggs for embryo transfer. We collected the eggs and cultured them overnight to eliminate infectious mycoplasma (Hill \& Stalley ,1991) prior to embryo transfer. Cleavage eggs that developed normally were transferred to five recipients. All five recipients became pregnant and 27 pups were born. Since the egg donors and male studs were both from the same mixed genetic background, all of the pups born carried the desired genotype. Because of the complex pathogen load in these mice, dams were sent to Charles River Laboratory for health assessment plus tests that showed the animals were pathogen free.

\section{Discussion}

We established a mouse pathogen rederivation program based on embryo transfer and show that it is effective for the elimination of mouse viral, bacterial, and parasitic infections. A total of 96 independent mouse strains were successfully rederived. Our embryo transfer procedures eliminated every mouse pathogen that we encountered and attempted to eradicate: MHV, MPV, EDIM, TMEV (GDVII), MAD, Helicobacter spp., Mycoplasma pulmonis, pinworm and fur mites. Although this is not a comprehensive list of all mouse pathogens, we eliminated three categories of organisms: viruses, bacteria, and parasites. It is likely that the application of embryo transfer to the rederivation of other mouse pathogens, such as protozoa, will be equally successful. Embryo transfer eliminates organisms from the environment of the preimplantation embryo by extensive washing in sterile media. Consequently, the pups that are born have the same health status as their surrogate mothers. In agreement with this, it was demonstrated that germ free mouse lines can be successfully derived by embryo transfer to germ free surrogate mothers (Okamoto \& Matsumoto, 1999). We conclude that embryo transfer is an efficient method for the pathogen free rederivation of infected mouse lines.

We observed that in all embryo transfer recipients, pathogens were eliminated whether egg donors were obtained from an enzootically infected founder colony or imported from a commercial vendor. When we mated heterozygous transgenic or gene-targeted stud males to wild type egg donors, we expected half the pups to have the desired genotype. In the successful rederivation procedures, we observed that 857 (43\%) of the 1996 pups born carried the desired genotype. This is fewer than expected $\left(p<0.005, \chi^{2}\right.$ Test). The reasons for this lower than expected frequency of pups with the desired genotype is unclear. It may the result of technical problems (insensitive genotyping assays) or the reduced viability of genetically engineered pups.

In addition to the 96 successful rederivations, we were unable to rederive 15 mouse lines. These were characterized by the failure to identify rederived pups of the desired genotype and not by the failure to eliminate pathogens. Factors that reduced the efficiency of embryo transfer rederivation in the 15 unsuccessful attempts can be divided into logistical problems: e.g. only one stud male available for embryo production and biological problems, e.g. inefficient production of fertilized eggs for embryo transfer. The principle difference between successful and unsuccessful rederivation procedures was the availability of four 
or more stud males with good reproductive performance. On several occasions, replacement of stud males older than 6 months with young animals changed unsuccessful attempts to successful ones. Initial compliance by laboratories to provide 4-6 stud males in prime reproductive condition minimized the time to complete successful rederivations. Of the 15 rederivations that did not produce pups with the desired genotype, nine depended on a single stud male, three depended on old males ( $>8$ months) and three others depended on the use of strains that did not produced fertilized eggs in response to superovulation and stud mating (one mixed background and DBA/2). Another possible factor is the sensitivity and accuracy of genotyping assays, which we did not perform and thus are unable to assess. Similarly we are unable to directly assess the effects of genetic manipulations on prenatal viability of the transferred embryos. Additional studies are necessary to address this issue.

Factors that affect the success of embryo transfer can be divided into three areas: (1) technical competency in surgery, (2) production of fertilized eggs for transfer, (3) selection of surrogate mothers and appropriate husbandry, and (4) the sensitivity of genotyping assays to detect the desired genotype. The first, third, and fourth factors are easily controlled by providing training to staff and by selecting outbred or vigorous hybrid female recipients. The single most critical factor in post-surgery husbandry to maintain pregnancy and establish lactation is provision of appropriate rodent diet, containing $6.0-6.5 \%$ fat. Pregnancy and post-partum survival is severely reduced in surrogate dams fed diet containing $4.5 \%$ fat (T. Saunders, unpublished observations). The second factor, production of fertilized eggs, depends on biological factors that are not always under control of the investigator. For example, some inbred strains respond poorly to superovulation treatments (Spearow, 1988) and produce few eggs for fertilization. Other inbred strains, such as PL/J have poor sperm quality that affect numbers of fertilized eggs (Pyle \& Handel, 2003; T. Saunders, unpublished observations). A less quantifiable factor is embryo sensitivity to experimental manipulation. In particular, DBA/2 rederivation is consistently difficult due to low birth rates after embryo transfer (Table 4 and unpublished observations). Trans- genic offspring are generally identified by PCR reactions that detect unique DNA sequences in the transgene. Our experience with numerous research laboratories indicates that genotype assignations are conservative, designed to exclude borderline results and to accept only robust positive reactions. Routine fluctuations in PCR assays can affect genotyping accuracy, particularly in the absence of rigorous quality control procedures. Factors that affect genotyping reproducibility include: (1) extraction of pure DNA from tail tip biopsies, (2) determination of the limit of PCR sensitivity (does the assay detect transgene DNA with single copy gene equivalency?), (3) appropriate positive and negative controls to demonstrate that mouse DNA is present in the assay, and (4) the absence of transgene DNA contamination. The majority of mouse rederivations proceed smoothly and successfully when all of these variables are controlled.

In the few cases, where mice are not rederived by embryo transfer the most common problem is obtaining fertilized eggs for embryo transfer. Often this is resolved by increasing the number of stud males to four and/or replacing old stud males with animals in their reproductive prime (2-6 months old). If this does not result in fertilized eggs then alternative methods to obtain fertilized eggs include: in vitro fertilization (IVF), natural mating, and intracytoplasmic sperm injection (ICSI). IVF procedures can yield hundreds of fertilized eggs for embryo transfer from a single procedure with sperm pooled from a few males (Thornton et al., 1999). Suzuki et al. (1996) successfully eliminated MHV and Pasteurella pneumotropica with IVF followed by embryo transfer. Fewer eggs are produced by natural mating than by superovulation. The need for several females to ovulate spontaneously at the same time increases the number of animals required for fertilized egg production by natural mating. If natural mating is not effective due to low sperm counts then ICSI can be used because few individual sperm are needed to produce fertilized eggs by this method. This approach may not be suitable when specific genetic backgrounds must be rederived because ICSI is significantly less efficient in inbred strains than in hybrid strains (Kawase et al., 2001). The alternative to rederivation by embryo transfer is rederivation by hysterectomy. This approach can be used with strains of very low male and female fertility or for 
mouse embryos that do not survive well in culture. Hysterectomy relies on the production of synchronized pregnancies between the strain undergoing rederivation and an SPF mouse colony that is used for fostering pups. The disadvantages include the loss of viable pups during hysterectomy, the increased probability of infecting pups or surrogate mothers during the procedure, the elimination of pups from the SPF litter, and the loss of pups ignored by SPF surrogates. Although animalintensive hysterectomy may be indicated when other rederivation procedures are ineffective.

In the context of animal welfare principles (Flecknell, 2002) pathogen rederivation offers refinement of experimental design by reducing pain and distress. It can be anticipated that healthy, SPF mice will more faithfully reflect physiological changes due to genetic alterations than diseased mice. This, in turn, can reduce variability in experimental groups and the number of animals needed to draw statistically meaningful conclusions. In comparison to other methods, embryo transfer rederivation reduces the number of animals required for rederivation. In the average rederivation procedure, we used four superovulated egg donors. IVF and ICSI procedures often require 10-20 females per procedure. Hysterectomy requires multiple synchronized litters of infected and SPF mice. Some pups from infected litters do not survive the procedure and pups are lost from SPF litters when they are replaced by rederivation pups. As a consequence, hysterectomy is the most animal intensive rederivation procedure.

Embryo transfer is an effective method to eliminate pathogens from genetically engineered mouse strains. The study of pathogen free animals eliminates the confounding effects of pathogen infection from research results. Further studies are needed to determine if lower than expected transmission ratios of transgenes and mutant alleles are due to inaccurate genotyping assays or reduced embryo survival. Future directions include the wider application of this method to additional pathogens and to the establishment of germ free mouse strains.

\section{Acknowledgments}

We thank Steve Brown, David Burke, Margaret Burmeister, Sally Camper, Jeffrey Chamberlain,
Wes Dunnick, Bill Fay, Eric Fearon, David Ginsburg, Jerry Gorski, David Kohrman, Andy Griffith, Tom Hornyak, Laurie McCauley, Miriam Meisler, Joe Metzger, Diane Robins and Audrey Seasholtz for providing mice for rederivation. We thank Tina Hutchinson and Corey Ziebell for their help managing the mouse colonies. We thank Robert Dysko, Claire Hankenson, Daniel Ringler, and Howard Rush for helpful discussions. We thank Sally Camper and Linda Samuelson for their critical review of the manuscript. The Transgenic Core receives support from the University of Michigan Comprehensive Cancer Center, University of Michigan Rheumatic Diseases Center Core, the University of Michigan Center for Organogenesis, and the University of Michigan Gut Peptide Research Center.

\section{References}

Abzug MJ and Tyson RW (2000) Protection of murine gestational tissues from picornavirus infection in the preimplantation period. Placenta 21: 422-426.

Carthew P, Wood MJ and Kirby C (1983) Elimination of Sendai (parainfluenza type 1) virus infection from mice by embryo transfer. J Reprod Fertil 69: 253-257.

Carthew P, Wood MJ and Kirby C (1985) Pathogenicity of mouse hepatitis virus for preimplantation mouse embryos. J Reprod Fertil 73: 207-213.

Colombo LL, Stazzone A, Chen GJ, Lopez MC and Watson RR (2000) Behavioral and immune changes in v-Ha-ras transgenic mice Immunopharm. Immunotox 22: 339-356.

Davis JA, Paylor R, McDonald MP, Libbey M, Ligler A, Bryant K et al. (1999) Behavioral effects of Ivermectin in mice. Lab Anim Sci 49: 288-296.

De Rijk EP, Van Rijk AF, Van Esch E, De Jong WW, Wesseling P and Bloemendal H (2000) Demyelination and axonal dystrophy in alpha A-crystallin transgenic mice. Int $J$ Exp Path 81: 271-282.

Engle SJ, Ormsby I, Pawlowski S, Bolvin GP, Corft J, Balish E et al. (2002) Elimination of colon cancer in germ-free transforming growth factor beta 1-deficient mice. Cancer Res 62: 6362-6366.

Flecknell P (2002) Replacement, reduction and refinement. ALTEX 19:73-78.

Glass WG, Chen BP, Liu MT and Lane TE (2002) Mouse Hepatitis Virus infection of the central nervous system: Chemokine-mediated regulation of host defense and disease. Viral Immun 15: 261-272.

Hill AC and Stalley GP (1991) Mycoplasma pulmonis infection with regard to embryo freezing and hysterectomy derivation. Lab Anim Sci 41: 353-356.

Hogan B, Beddington R, Constantini F and Lacy E (1994) Manipulating the Mouse Embryo: A Laboratory Manual. Cold Spring Harbor Press, New York.

Homberger FR (1997) Enterotropic mouse hepatitis virus. Lab Animals 31: 97-115. 
Homberger FR, Ilgen-Wicke B, Jacobi K, Kraft V, Kunstyr I, Mähler M et al. (1999) Implications of infectious agents on results of animal experiments. Lab Animals 33, Supplement 1: 39-87.

Jacoby RO, Ball-Goodrich LJ, Besselsen DG, McKisic MD, Riley LK and Smith AL (1996) Rodent parvovirus infection. Lab Anim Sci 46: 370-380.

Kawase Y, Iwata T, Toyoda Y and Wakayama T (2001) Comparison of intracytoplasmic sperm injection for inbred and hybrid mice. Mol Reprod Develop 60: 74-78.

Mohanty SB and Bachman PA (1974) Susceptibility of fertilized mouse eggs to minute virus of mice. Infect Immun 9 : 762-763.

Morrell JM (1999) Techniques of embryo transfer and facility decontamination used to improve the health and welfare of transgenic mice. Lab Animals 33: 201-216.

National Research Council (1991) Infectious Diseases of Mice and Rats. National Academy Press, Washington, D.C. USA.

Okamoto M and Matsumoto T (1999) Production of germfree mice by embryo transfer. Exp Anim 48: 59-62.

Pyle A and Handel MA (2003) Meiosis in male PL/J mice: a genetic model for gametic aneuploidy. Mol Reprod Dev 64: $471-481$.

Reetz IC, Wullenweber-Schmidt M, Kraft V and Hedrich, HJ (1988) Rederivation of inbred strains of mice by means of embryo transfer. Lab Anim Sci 38: 696-701.
Shi L, Fatemi SH, Sidwell RW and Patterson PH (2003) Maternal influenza infection causes marked behavioral and pharmacological changes in the offspring. $J$ Neurosci 23: 297-302.

Spearow JL (1988) Major genes control hormone-induced ovulation rate in mice. $J$ Reprod Fertil 82: 787-797.

Stringfellow DA (1998) Recommendations for the sanitary handling of in-vivo-derived embryos. In: Stringfellow DA and Seidel SM (eds), Manual of the International Embryo Transfer Society. (pp. 79-91)Savoy, IL.

Suzuki H, Yorozu K, Watanabe T, Nakura M, and Adachi J (1996) Rederivation of mice by means of in vitro fertilization and embryo transfer. Exp Anim 45:33-38.

Thornton CE, Brown, SDM, and Glenister PH (1999) Large numbers of mice established by in vitro fertilization with cryopreserved spermatozoa: implications and applications for genetic resource banks, mutagenesis screens, and mouse backcrosses. Mamm. Genome 10: 987-992.

Tsunoda I, Iwasaki Y, Terunuma H, Sako K and Ohara Y (1996) A comparative study of acute and chronic diseases induced by two subgroups of Theiler's murine encephalomyelitis virus. Acta Neuropath 91: 595-602.

Zeller W, Meier G, Bürki K and Panoussis B (1998) Adverse effects of tribromoethanol as used in the production of transgenic mice. Lab Animals 32: 407-413. 